\title{
0 antagonismo com acetamida em experimentos com ovinos, caprinos e coelhos indica monofluoroacetato como princípio tóxico de Pseudocalymma elegans Bignoniaceae ${ }^{1}$
}

\author{
Michel A. Helayel ${ }^{2}$, Saulo A. Caldas ${ }^{2}$, Tiago C. Peixoto ${ }^{2}$, Ticiana N. França ${ }^{3}$, Carlos H. \\ Tokarnia $^{4}$, Jürgen Döbereiner ${ }^{5}$, Vivian A. Nogueira ${ }^{3}$ e Paulo V. Peixoto ${ }^{4}$
}

\begin{abstract}
Helayel M.A., Caldas S.A., Peixoto T.C., França, T.N., Tokarnia C.H., Döbereiner J., Nogueira V.A. \& Peixoto P.V. 2011. [Antagonism of acetamid in experiments with sheep, goats and rabbits indicates that monofluoroacetate is the toxic principle of Pseudocalymma elegans Bignoniaceae.] 0 antagonismo com acetamida em experimentos com ovinos, caprinos e coelhos indica monofluoroacetato como princípio tóxico de Pseudocalymma elegans Bignoniaceae. Pesquisa Veterinária Brasileira 31(10):867-874. Projeto Sanidade Animal Embrapa/UFRRJ, Seropédica, RJ 23890-000, Brazil. E-mail: michel_abdallavet@yahoo.com.br

This study aimed to evaluate the protective effect of acetamid in experimental poisoning by Pseudocalymma elegans in sheep, goats and rabbits, in order to prove indirectly that monofluoroacetate (MF) is responsible for the clinical signs and death of animals that ingested the plant. Experiments were performed to determine for sheep and goats the lethal dose of P. elegans collected in Rio Bonito, RJ, in different seasons, and to adjust the dose of acetamid to be administered. - In the first experiment, two sheep and two goats received $1.0 \mathrm{~g} / \mathrm{kg}$ of fresh P. elegans, and two (one sheep and one goat) were pretreated with $2.0 \mathrm{~g} / \mathrm{kg}$ of acetamid. None of the animals showed clinical signs or died. Possibly, the plant could be less toxic, since it was collected at the end of the rainy season. - In the second experiment, two sheep and two goats received 0.67 and $1.0 \mathrm{~g} / \mathrm{kg}$ of the dried plant, after pretreatment with 2.0 and $3.0 \mathrm{~g} / \mathrm{kg}$ of acetamid, respectively. All animals died, as the administered doses of $P$. elegans were very high. - In the third experiment, two sheep and two goats received $0.333 \mathrm{~g} / \mathrm{kg}$ of dried P. elegans after previous administration of $2.0 \mathrm{~g} / \mathrm{kg}$ of acetamid; a week later, the protocol above was repeated, but without the antidote. In experiments with rabbits, doses of 0.5 and $1.0 \mathrm{~g} / \mathrm{kg}$ of dried P. elegans were given after administration of $3.0 \mathrm{~g} / \mathrm{kg}$ of acetamid; seven days later, the same protocol was repeated, except the administration of acetamide. This procedure, when acetamid was administered before, prevented the appearance of clinical signs and death of sheep, goats and rabbits. But the animals not treated with acetamid showed symptoms of poisoning and died. Clinically, the sheep and goats had tachycardia, engorged jugular vein, positive venous pulse, lateral recumbence, and muscle tremors. In the "dramatic phase", the animals fell into lateral position, stretched the limbs, were paddling and died within minutes. The rabbits showed apathy, muscle tremors, vocalization and lateral decumbence minutes before death. At postmortem examination, the sheep and goats had engorged jugular veins and atria, dilated vena cava cranialis and caudalis, as well as pulmonary edema, hepatic congestion and edema of the gallbladder subserosa. In rabbits, the main macroscopic alterations were dilated atria, engorged vena
\end{abstract}

\footnotetext{
1 Recebido em 10 de dezembro de 2010.

Aceito para publicação em 29 de junho de 2011.

Parte da Tese de Doutorado do primeiro autor, defendida na c, Seropédica, RJ 23890-000, Brasil.

${ }^{2}$ Curso de Pós-Graduação em Ciências Veterinárias, Instituto de Veterinária, Universidade Federal Rural do Rio de Janeiro (UFRRJ), Seropédica, RJ 23890-000. *Autor para correspondência: Michel_abdallavet@yahoo.com.br
}

\footnotetext{
${ }^{3}$ Departamento Epidemiologia e Saúde Pública, Instituto de Veterinária, Universidade Federal Rural do Rio de Janeiro (UFRRJ), Seropédica, RJ 23890000.

${ }^{4}$ Departamento de Nutrição Animal e Pastagem, Instituto de Zootecnia, Universidade Federal Rural do Rio de Janeiro (UFRRJ), Seropédica, RJ 23890000 .

${ }^{5}$ Projeto Sanidade Animal Embrapa/UFRRJ, Seropédica, RJ.
} 
cava cranialis and caudalis, and congested liver and diaphragm vessels. Histopathology revealed, in two sheep and one goat, vacuolar-hydropic degeneration of the distal convoluted kidney tubules, together with caryopicnosis. In the rabbits, the liver showed severe congestion with numerous shock corpuscles. The experimental results show indirectly that MF is to be held responsible for death of the animals that ingested P. elegans; since "acetate donor" compounds, such as acetamid, are capable to reduce the competitive inhibition of MF for the same active site (Coenzyme A) which prevents the formation of fluorocitrate, its active metabolite, formed in the body through the so-called "lethal synthesis".

INDEX TERMS: Pseudocalymma elegans, acetamide, poisonous plants, pathology.

RESUMO.- 0 presente trabalho teve como objetivo avaliar o efeito protetor da acetamida nas intoxicações experimentais por Pseudocalymma elegans em ovinos, caprinos e coelhos, com a finalidade de comprovar indiretamente que o monofluoroacetato (MF) é responsável pelos sinais clínicos e a morte dos animais que ingerem essa planta. Foram realizados experimentos para determinar a dose letal da planta coletada em Rio Bonito, RJ, em diferentes épocas do ano para ovinos e caprinos e ajustar a dose de acetamida a ser administrada. - No primeiro experimento, dois ovinos e dois caprinos receberam $1,0 \mathrm{~g} / \mathrm{kg}$ de P. elegans fresca e um animal de cada espécie foi tratado previamente com $2,0 \mathrm{~g} / \mathrm{kg}$ de acetamida. Nenhum animal apresentou alterações clínicas ou morreu. Ao que tudo indica a planta poderia estar menos tóxica, já que foi coletada no fim da estação das águas. - No segundo experimento, dois ovinos e dois caprinos receberam 0,67 e $1,0 \mathrm{~g} / \mathrm{kg}$ da planta dessecada, após tratamento prévio, com 2,0 e 3,0g/ $\mathrm{kg}$ de acetamida, respectivamente. Todos os animais morreram, pois administramos doses muito altas de P. elegans. - No terceiro experimento, dois ovinos e dois caprinos receberam $0,333 \mathrm{~g} / \mathrm{kg}$ de $P$. elegans dessecada, após administração prévia de $2,0 \mathrm{~g} / \mathrm{kg}$ de acetamida. Uma semana depois, o protocolo acima foi repetido, porém sem o antídoto. Nos experimentos com coelhos, foram administradas doses de 0,5 e $1,0 \mathrm{~g} / \mathrm{kg}$ de P. elegans dessecada após a administração de $3,0 \mathrm{~g} / \mathrm{kg}$ de acetamida. Sete dias depois, repetiu-se o protocolo, com exceção da administração de acetamida. Esta, quando administrada previamente, evitou o aparecimento dos sinais clínicos e a morte dos ovinos, caprinos e coelhos, já os animais não tratados com acetamida apresentaram sintomatologia e morreram. Clinicamente, os ovinos e caprinos manifestaram taquicardia, jugulares ingurgitadas, pulso venoso positivo, decúbito esternal e tremores musculares. Na "fase dramática", os animais caíam em decúbito lateral, esticavam os membros, faziam movimentos de pedalagem e morriam em poucos minutos. Nos coelhos observaram-se apatia, tremores musculares, decúbito lateral e vocalização minutos antes da morte. A avaliação macroscópica revelou, nos ovinos e caprinos, jugulares ingurgitadas, aurículas, veia cava caudal e cranial dilatadas, além de edema pulmonar, congestão hepática e edema na subserosa da vesícula biliar. Nos coelhos as principais alterações observadas foram aurículas dilatadas, veia cava caudal e cranial ingurgitadas, fígado e vasos do diafragma congestos. 0 exame histopatológico revelou, em dois ovinos e um caprino, degeneração hidrópico-vacuolar dos túbulos uriníferos contornados distais associada à cariopicnose. Nos coelhos havia congestão hepática acentuada com numerosos corpúsculos de choque. Nossos resultados comprovam, de forma indireta, que MF é responsável pela morte dos animais que ingerem essa planta, uma vez que compostos "doadores de acetato" como a acetamida, são capazes de reduzir a inibição competitiva do MF pelo mesmo sítio ativo (Coenzima A), o que impede a formação do fluorocitrato, seu metabólito ativo, formado no organismo por meio da denominada "síntese letal".

TERMOS DE INDEXAÇÃO: Pseudocalymma elegans, acetamida, plantas tóxicas, patologia.

\section{INTRODUÇÃO}

No Brasil, as plantas que causam "morte súbita" são consideradas as mais importantes, pelo menos no que diz respeito aos prejuízos econômicos. A principal planta desse grupo é Palicourea marcgravii, responsável pela grande maioria das mortes de bovinos intoxicados por plantas no território nacional. Outras plantas capazes de causar "morte súbita" têm distribuição geográfica mais limitada, como é o caso da Pseudocalymma elegans, que só está presente no Estado do Rio de Janeiro. Em geral, os animais que ingerem essas plantas morrem sem sinais clínicos prévios, ou com sintomas que passam despercebidos; à necropsia não se encontram lesões significativas. Pelo exame histológico, porém, em muitos animais encontra-se, no rim, uma lesão considerada característica, denominada "degeneração hidrópico-vacuolar das células epiteliais dos túbulos uriníferos contornados distais associada à cariopicnose" (Tokarnia et al. 2000).

0 monofluoroacetato (MF) tem sido isolado de diversas plantas tóxicas, cuja ingestão determina "morte súbita" na África do Sul e na Austrália; no Brasil, essa substância foi identificada em P. marcgravii por Oliveira (1963) e Arrabidaea bilabiata (Krebs et al. 1994); há ainda indícios da presença do MF em Mascagnia rigida (Cunha 2008). Recentemente, Nogueira et al. (2010) e Peixoto et al. (2010) demonstraram que bovinos e ovinos intoxicados experimentalmente com monofluoroacetato de sódio desenvolvem a degeneração hidrópico- vacuolar das células epiteliais dos túbulos uriníferos contornados distais associada à cariopicnose. Embora o quadro clínico-patológico verificado em animais que ingerem essas plantas seja semelhante, em muitos aspectos, ao observado nos casos de intoxicação por MF 
(Nogueira et al. 2010, Peixoto et al. 2010, Peixoto et al. 2011a), alguns pesquisadores são da opinião de que esse composto não seria responsável pela morte dos animais que ingerem essas plantas ou que outras substâncias causariam a morte dos animais ou que ainda poderiam contribuir para a toxicidade dessas plantas.

0 presente trabalho teve como objetivo avaliar o quadro clínico-patológico da intoxicação por P. elegans em ovinos, caprinos e coelhos com e sem a administração de acetamida, que é um composto doador de acetato utilizado como antídoto nos casos de intoxicação por MF. Pretende-se, dessa forma, comprovar indiretamente que o MF é responsável pela morte dos animais que ingerem essa planta, o que abriria boas possibilidades de estabelecerem-se medidas profiláticas e de tratamento.

\section{MATERIAL E MÉTODOS}

Os experimentos com a brotação de Pseudocalymma elegans (Vell.) Kuhlm. (fam. Bignoniaceae) foram realizados no Setor de Anatomia Patológica, do Projeto Sanidade Animal, Embrapa/UFRRJ, Seropédica, RJ. Utilizaram-se seis ovinos, seis caprinos mestiços e três coelhos, de ambos os sexos, clinicamente sadios.

Ovinos. No primeiro experimento, os Ovinos 1 e 2 receberam, por via oral, $1 \mathrm{~g} / \mathrm{kg}$ de brotos (roxos) recém-coletados de P. elegans. Ovino 1 recebeu ainda $2,0 \mathrm{~g} / \mathrm{kg}$ de acetamida, antes da administração da planta. 0 segundo experimento foi constituído de duas etapas; na primeira, os Ovinos 5 e 6 receberam, respectivamente, através de sonda orogástrica, $0,67 \mathrm{~g} / \mathrm{kg}$ e $1,0 \mathrm{~g} / \mathrm{kg}$, de brotos (roxos) dessecados, triturados e suspendidos em $50 \mathrm{~mL}$ de água. Os animais foram tratados previamente com $2,0 \mathrm{~g} / \mathrm{kg}$ e $3,0 \mathrm{~g} / \mathrm{kg}$ de acetamida, respectivamente. Na segunda etapa (uma semana depois), a mesma quantidade de $P$. elegans foi administrada aos animais, porém sem acetamida. 0 terceiro experimento também foi realizado em duas etapas; na primeira administraram-se, por via orogástrica, aos Ovinos 9 e $10,0,333 \mathrm{~g} / \mathrm{kg}$ de brotos (roxos) de P. elegans dessecados, triturados e suspendidos em $50 \mathrm{~mL}$ de água. Estes ovinos receberam tratamento prévio com $2,0 \mathrm{~g} / \mathrm{kg}$ de acetamida. Na segunda etapa (uma semana depois), administrou-se apenas a mesma dose $(0,333 \mathrm{~g} / \mathrm{kg})$ da planta aos Ovinos 9 e 10 .

Caprinos. No primeiro experimento, os Caprinos 3 e 4 receberam, por via oral, $1,0 \mathrm{~g} / \mathrm{kg}$ de brotos (roxos) recém- coletados de P. elegans. 0 Caprino 3 recebeu ainda 2,0g/kg de acetamida, antes da administração da planta. 0 segundo experimento foi constituído de duas etapas; na primeira, os Caprinos 7 e 8 receberam, respectivamente, por via orogástrica, $0,67 \mathrm{~g} / \mathrm{kg}$ e $1,0 \mathrm{~g} / \mathrm{kg}$, de brotos (roxos) de P. elegans dessecados, triturados e suspendidos em $50 \mathrm{~mL}$ de água. Os Caprinos 7 e 8 receberam antes $2,0 \mathrm{~g} / \mathrm{kg}$ e 3,0g/kg de acetamida, respectivamente. Na segunda etapa (uma semana depois) administrou-se esta mesma quantidade da planta, porém sem acetamida. 0 terceiro experimento também foi realizado em duas etapas; na primeira, os Caprinos 11 e 12 receberam, por via orogástrica, $0,333 \mathrm{~g} / \mathrm{kg}$ de brotos (roxos) dessecados, triturados e suspendidos em $50 \mathrm{~mL}$ de água. Estes caprinos tinham sido tratados previamente com $2,0 \mathrm{~g} / \mathrm{kg}$ de acetamida. $\mathrm{Na}$ segunda etapa (realizada uma semana depois), administrou-se a apenas a mesma quantidade $(0,333 \mathrm{~g} / \mathrm{kg})$ de planta a estes caprinos.
Administração da acetamida: em todos os experimentos com ovinos e caprinos, a acetamida foi diluída em $100 \mathrm{~mL}$ de água e a solução foi dividida em duas partes iguais; a primeira parte foi administrada 4 horas e a segunda 2 horas antes da administração da planta.

Coelhos. Três coelhos receberam por via intragástrica, respectivamente, doses de 1,$0 ; 0,5$ e $0,5 \mathrm{~g} / \mathrm{kg}$ de brotos (roxos) de P. elegans previamente dessecados, triturados e suspendidos em $50 \mathrm{~mL}$ de água. Os animais receberam tratamento prévio com $3,0 \mathrm{~g} / \mathrm{kg}$ acetamida diluída em $25 \mathrm{ml}$ de água, 2 horas antes da administração da planta.

Todas as necropsias nos ovinos, caprinos e coelhos foram feitas imediatamente após a morte dos animais. Fragmentos de diversos órgãos foram fixados em formol a $10 \%$, processados rotineiramente para histopatologia, corados pela Hematoxilina e Eosina.

\section{RESULTADOS}

Os principais resultados referentes ao quadro clínico-patológico e o desfecho são apresentados no Quadro 1.

Início dos sinais clínicos e duração dos sintomas graves ("fase dramática")

Ovinos. No primeiro experimento com os brotos recém-coletados de Pseudocalymma elegans, os Ovinos $1 \mathrm{e}$ $2(1,0 \mathrm{~g} / \mathrm{kg})$ não apresentaram sinais clínicos da intoxicação. No segundo experimento com os brotos dessecados, o Ovino $6(1,0 \mathrm{~g} / \mathrm{kg})$ morreu $12 \mathrm{~h} 54 \mathrm{~min}$ após o início da sintomatologia. Ovino $5(0,67 \mathrm{~g} / \mathrm{kg})$ foi encontrado morto. Na primeira etapa do terceiro experimento, os Ovinos 9 e $10(0,333 \mathrm{~g} / \mathrm{kg}$ da planta dessecada) não apresentaram sintomatologia; já na segunda etapa, os sintomas apareceram 3h1min e 3h36min após a administração da planta e a morte sobreveio em 3 e 5 minutos após o início da sintomatologia, respectivamente. (Quadro 1)

Caprinos. No primeiro experimento, os Caprinos 3 e $4(1,0 \mathrm{~g} / \mathrm{kg})$ não apresentaram sinais clínicos da intoxicação. No segundo experimento, o Caprino $7(0,67 \mathrm{~g} / \mathrm{kg})$ apresentou os primeiros sinais clínicos $28 \mathrm{~h} 27 \mathrm{~min}$ após a administração da planta, morrendo após a fase dramática de 8 minutos. 0 Caprino $8(1,0 \mathrm{~g} / \mathrm{kg})$ apresentou os primeiros sintomas $4 \mathrm{~h}$ após a ingestão da planta e foi encontrado morto. Na primeira etapa do terceiro experimento, os Caprinos 11 e $12(0,333 \mathrm{~g} / \mathrm{kg}$ da planta dessecada) não apresentaram sintomatologia; já na segunda etapa, os sinais clínicos iniciaram-se $5 \mathrm{~h} 20 \mathrm{~min}$ e $6 \mathrm{~h} 4 \mathrm{~min}$ depois da administração da planta, e a morte sobreveio 2 minutos após. (Quadro 1)

Coelhos. Ao receberem previamente acetamida, os coelhos não apresentaram sinais clínicos da intoxicação (Quadro 1).

\section{Quadro clínico geral}

Nos ovinos e caprinos foram observados apatia, taquicardia, jugulares ingurgitadas (Fig.1), pulso venoso positivo, respiração abdominal, leve perda de equilíbrio, decúbito esternal às vezes com pescoço voltado para o flanco, ranger de dentes, movimentos mastigatórios, polaquiúria, inquietação e tremores musculares. Dois animais (Ovino 
Quadro 1. Resultados dos experimentos com Pseudocalymma elegans com e sem administração prévia de acetamida em ovinos, caprinos e coelhos

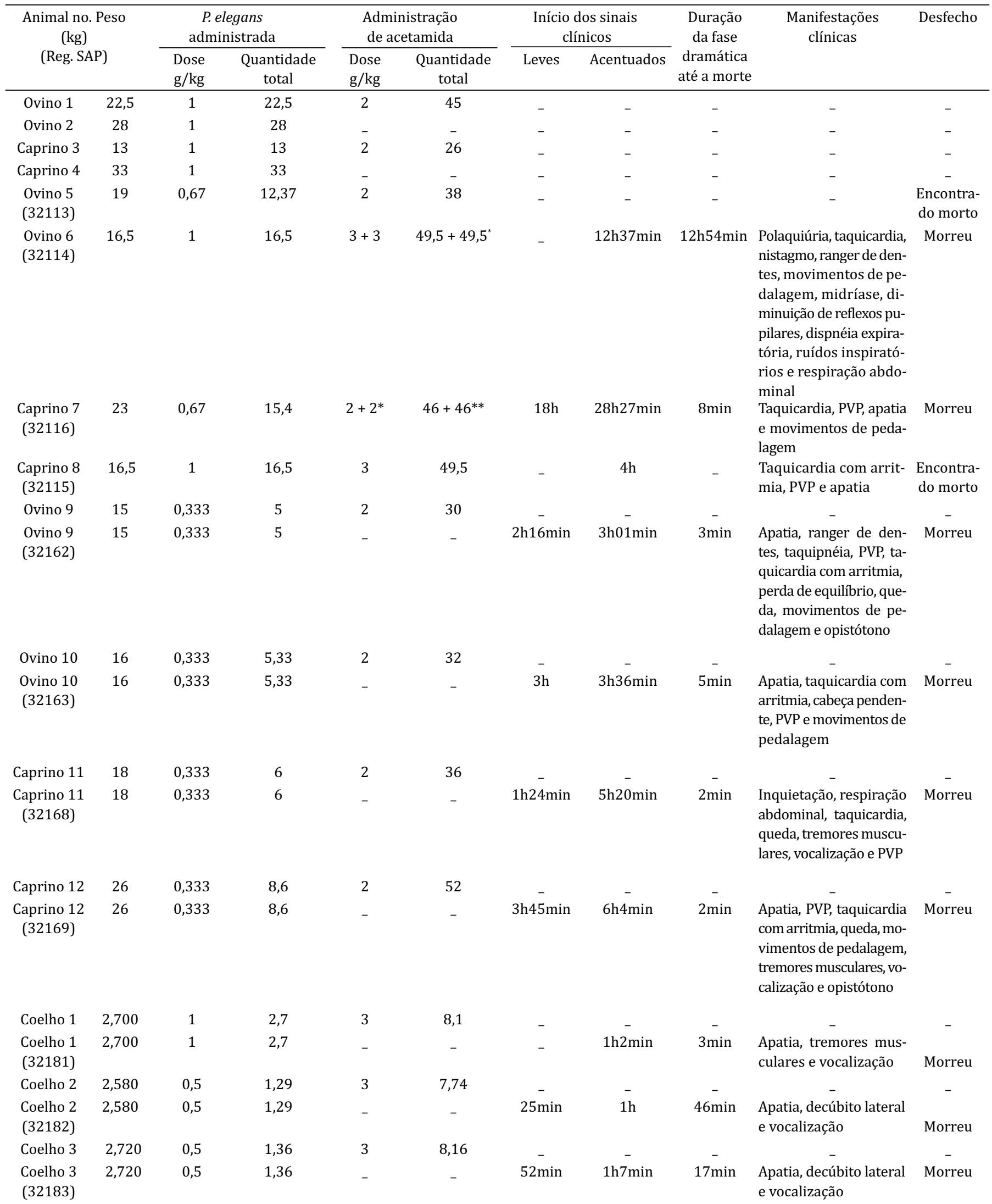

${ }^{*}$ Reforço de acetamida $19 \mathrm{~h}$ após a primeira dose. ${ }^{* *}$ Reforço de acetamida $23 \mathrm{~h} 30 \mathrm{~min}$ após a primeira dose. PVP = Pulso venoso positivo. 
6 e Caprino 7) apresentaram convulsão e três (Ovino 10 e Caprinos 8 e 12), arritmia. Pouco antes da "fase dramática", todos os animais deitavam-se e levantavam-se rapidamente várias vezes em curto espaço de tempo, caíam em decúbito lateral, esticavam os membros, faziam movimentos de pedalagem (Fig.2), apresentavam respiração ofegante, opistótono, nistagmo, mugidos e morriam em poucos minutos.

Nos coelhos observaram-se apatia, tremores musculares, decúbito lateral e vocalização minutos antes da morte. (Quadro 1).

\section{Achados de necropsia}

A avaliação macroscópica revelou, nos ovinos e caprinos, jugulares ingurgitadas, aurículas, veia cava caudal e cranial dilatadas e repletas de sangue (Fig.3). Nos Ovinos

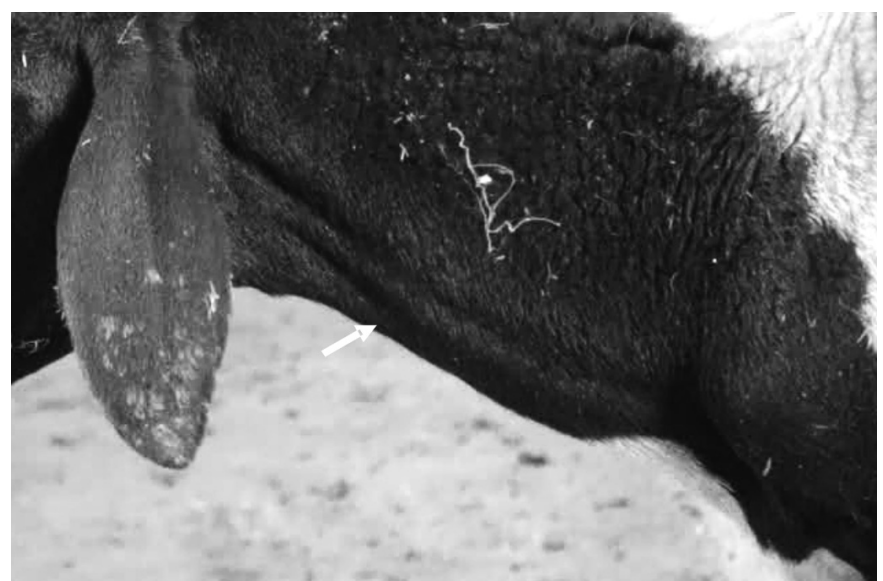

Fig.1. Ovino 9, que recebeu 0,333g/kg de Pseudocalymma elegans sem tratamento prévio com acetamida, com jugular ingurgitada (seta).

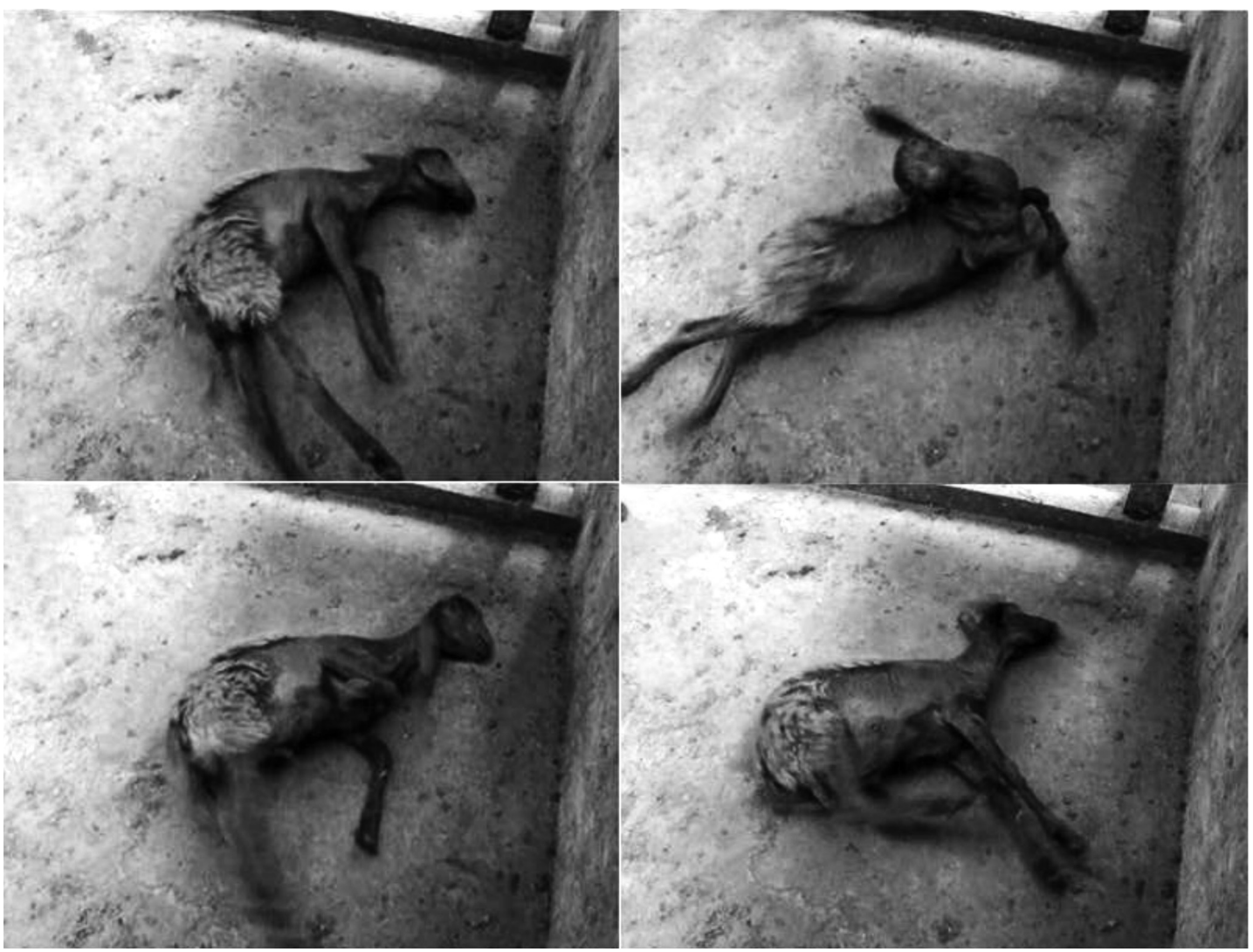

Fig.2. Ovino 10, que recebeu 0,333g/kg de Pseudocalymma elegans sem tratamento prévio com acetamida, em movimentos de pedalagem na "fase dramática".

5, 9 e 10 havia congestão dos vasos do diafragma e, no Ovino 5 , observaram-se ainda, vasos do pulmão dilatados. No Caprino 11 e no Ovino 9 havia grande quantidade de líquido espumoso de coloração avermelhada na traquéia e nos brônquios (Fig.4). Verificaram-se ainda congestão hepática em três animais (Ovinos 9, 10 e Caprino 11) e edema na inserção da parede da vesícula biliar ao fígado e no mesentério do Ovino 10. Nos coelhos as principais alterações foram aurículas dilatadas, veia cava caudal e cranial ingurgitadas e marcada congestão hepática.

\section{Histopatologia}

0 exame histopatológico revelou, no rim dos Ovinos 5 e 6 e do Caprino 7, moderada a acentuada degeneração hidrópico-vacuolar das células epiteliais dos túbulos uriníferos contornados distais (DHV) associada à cariopicnose (Fig.5). No fígado de todos os animais havia leve a moderada tumefação e vacuolização de hepatócitos, além de leve congestão no Caprino 12 e numerosos corpúsuclos de choque (Fig.6) e marcada congestão hepática nos três coelhos. Havia ainda leve a moderado edema pulmonar e moderada 


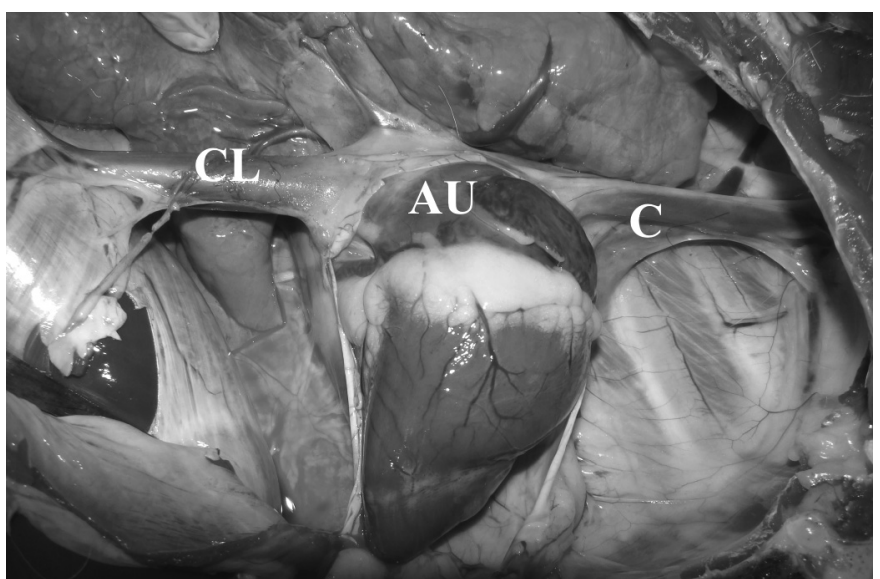

Fig.3. Aurícula (AU), veias cava caudal (CL) e cranial (C) repletas do Ovino 6 que recebeu 1g/kg de Pseudocalymma elegans.

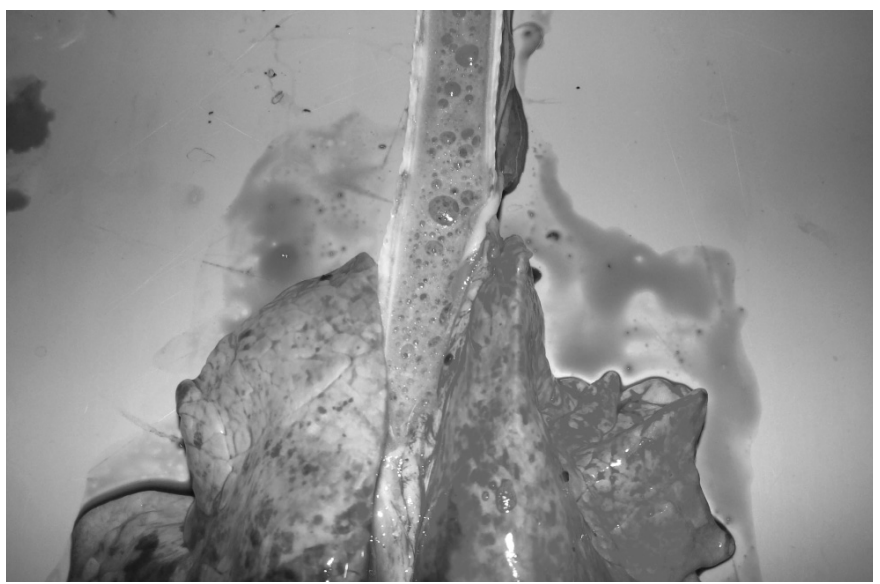

Fig.4. Acentuado edema pulmonar caracterizado por espuma rosada na traquéia do Caprino 11 que recebeu 0,333g/kg de Pseudocalymma elegans sem tratamento prévio com acetamida.

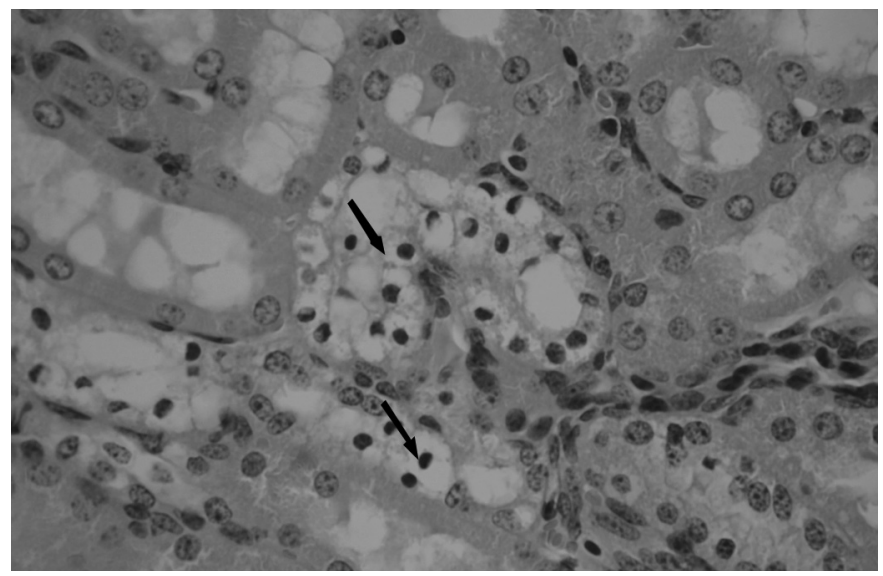

Fig.5. Degeneração hidrópico-vacuolar das células epiteliais dos túbulos uriníferos contornados distais associada à cariopicnose (setas) no Ovino 6 que recebeu 1,0g/kg de Pseudocalymma elegans. HE, obj. $25 \mathrm{x}$.

a acentuada tumefação no epitélio da bexiga de um coelho.

\section{DISCUSSÃO}

0 tratamento prévio com acetamida em ovinos e caprinos do terceiro experimento, bem como em coelhos intoxica-

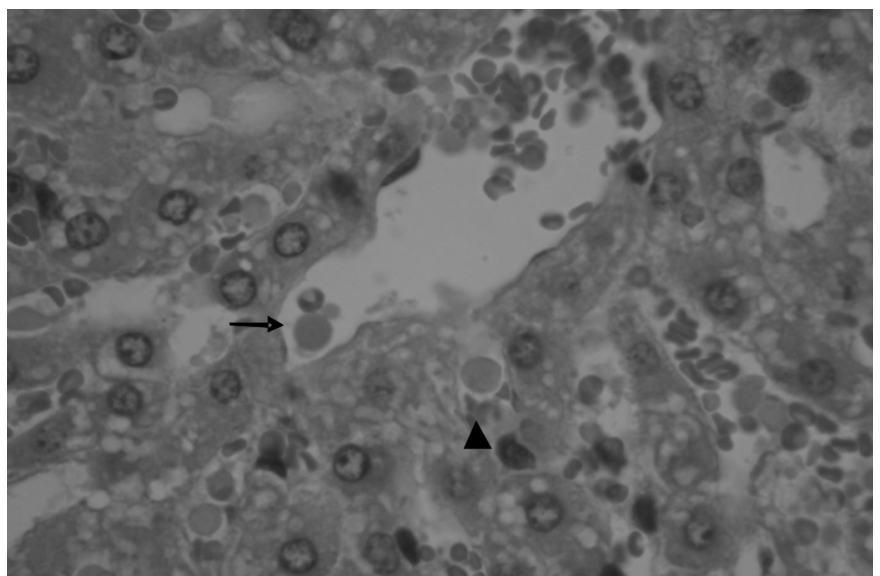

Fig.6. Corpúsculos de choque na veia centrolobular (seta) e nos sinusóides hepáticos (cabeça da seta), tumefação e vacuolização de hepatócitos no Coelho 1 que recebeu 0,5g/kg de Pseudocalymma elegans. HE, obj.40x.

dos por Pseudocalymma elegans, evitou o aparecimento dos sinais clínicos e o óbito com evolução superaguda, característicos da intoxicação por plantas que causam "morte súbita”. Isso indica que o monofluoroacetato é o princípio tóxico desta planta.

Esses resultados foram semelhantes aos descritos por Peixoto et al. (2011c), nos quais a administração anterior de acetamida a ratos que receberam folhas frescas de $\mathrm{Pa}$ licourea marcgravii, por extratos concentrados de $P$. marcgravii, P. juruana, Pseudocalymma elegans, Arrabidaea bilabiata, Mascagnia rigida, M. pubiflora e por MF, também impediu o óbito. A administração prévia de $2,0 \mathrm{~g} / \mathrm{kg}$ de acetamida aos bovinos que receberam $0,5 \mathrm{mg} / \mathrm{kg}$ de $\mathrm{MF}$ ou $1,0 \mathrm{~g} / \mathrm{kg}$ de P. marcgravii, também foi capaz de evitar o aparecimento dos sinais clínicos e a morte de todos os animais (Peixoto et al. 2011b). De fato, já em 1994, Górniak et al. relataram o bom efeito protetor da acetamida em relação à intoxicação experimental por P. marcgravii em ratos, entretanto esses autores não concluíram que MF seria o fator determinante da morte desses animais. Estudos recentes, por outro lado, demonstram que MF é capaz de induzir degeneração hidrópico-vacuolar dos túbulos contornados distais (DHV) de bovinos e ovinos intoxicados por essa substância (Nogueira et al. 2010, Peixoto et al. 2010, Peixoto et al. 2011a). 0 efeito protetor da acetamida evidenciado nesse estudo foi similar ao descrito primeiramente em 1986 por Egyed \& Schultz, que avaliaram a eficácia da administração do MF em cobaios e ovinos intoxicados experimentalmente por Dichapetalum cymosum, planta africana cujo princípio tóxico é MF. Esses autores concluíram que a eficácia da acetamida depende de diversos fatores, como a toxidez da planta em questão, tempo decorrido entre a intoxicação e fornecimento do antídoto, doses de acetamida e da planta administradas. Realmente, tudo indica que, no segundo experimento, a quantidade de acetamida foi insuficiente para antagonizar a quantidade de MF (presumivelmente muito elevada) contida nas amostras de P. elegans $(0,67$ e $1,0 \mathrm{~g} / \mathrm{kg}$ da planta dessecada). Possivelmente, houve uma variação progressiva na toxidez da planta, coletada em diferentes épocas do ano. Essa variação de toxidez já foi detectada por 
Döbereiner et al. (1984) e por Jabour et al. (2006) em experimentos com outra Bignoniaceae que causa "morte súbita" (A. bilabiata) em coelhos.

Nos experimentos com ovinos e caprinos, em geral, a evolução clínica da intoxicação foi mais longa do que a descrita por Tokarnia et al. (1993) e por Consorte et al. (1994), respectivamente. Da mesma forma observou-se certa variação no tempo decorrido entre a ingestão e o aparecimento dos primeiros sinais clínicos, em animais que receberam a mesma dose de P. elegans. A esse respeito, Eisler (1995) sugere que a variação na resposta individual ao MF pode ser atribuída à reduzida habilidade na conversão do fluoroacetato em fluorocitrato. Por outro lado, Goncharov et al. (2005) consideram que as diferenças de sensibilidade estão relacionadas à taxa metabólica do organismo, especificamente, do metabolismo oxidativo celular, que pode ou não favorecer a metabolização e a eliminação de substâncias tóxicas.

Clinicamente, os ovinos e caprinos apresentaram alterações similares às descritas em outros experimentos realizados com P. elegans em ovinos (Consorte et al. 1994), caprinos (Tokarnia et al. 1993), bovinos (Tokarnia et al. 1969, Helayel et al. 2009), coelhos (Tavares et al. 1974, Helayel et al. 2009) e cobaios (Tavares et al. 1974) bem como na intoxicação por MF em bovinos (Nogueira et al. 2010, Peixoto et al. 2011b) e ovinos (Peixoto et al. 2010).

A sintomatologia nos coelhos foi caracterizada por apatia, tremores musculares, decúbitos atípicos e vocalização minutos antes da morte, sintomas também já descritos na intoxicação por P. elegans (Tavares et al. 1974, Helayel et al. 2009) e por MF (Caldas, dados não-publicados) nessa espécie.

Macroscopicamente, os animais apresentaram alterações correlacionadas com insuficiência cardíaca, tais como veia cava caudal, cranial e jugulares ingurgitadas, aurículas dilatadas e repletas de sangue, congestão dos vasos do diafragma e dos pulmões, edema pulmonar e congestão hepática, achados já relatados na intoxicação por P. elegans em coelhos, bovinos (Helayel et al. 2009), ovinos (Consorte et al. 1994) e caprinos (Tokarnia et al. 1993). Alterações similares também foram descritas na intoxicação por MF nessas espécies (Nogueira et al. 2010, Peixoto et al. 2011a, Peixoto et al. 2011b). Um achado necroscópico interessante foi o edema na subserosa da vesícula biliar e do mesentério, nunca observado em casos intoxicação por P.elegans, mas descrito nas intoxicações por MF, P.marcgravii (Nogueira et al. 2010, Peixoto et al. 2011b) e A. exotropica (Gava et al.1998a) em bovinos.

No presente estudo, o exame histológico revelou, em dois ovinos e em um caprino, o aparecimento da típica degeneração hidrópico-vacuolar dos túbulos uriníferos contornados distais associada à cariopicnose, lesão idêntica à descrita em 1959 por Döbereiner \& Tokarnia no rim de bovinos intoxicados por P. marcgravii, em ovinos intoxicados por P. elegans (Consorte et al., 1994). Mais tarde esta lesão também foi observada no rim de bovinos intoxicados natural e experimentalmente com todas as outras plantas brasileiras que causam "morte súbita" (Tokarnia et al. 2000, Barbosa et al. 2003, Oliveira et al. 2004, Helayel et al. 2009) e recentemente na intoxicação por MF em bovinos (Nogueira et al. 2010), ovinos (Peixoto et al. 2010) e ratos (Peixoto et al. 2011c).

O diagnóstico diferencial entre a intoxicação por P. elegans e demais plantas que causam morte súbita deve ser feito, sobretudo, com base nos dados epidemiológicos, uma vez que nenhuma outra planta desse grupo se desenvolve no habitat da P. elegans (Tokarnia et al. 2000). Devemos lembrar, entretanto que, ao contrário do que ocorre em bovinos, a intoxicação de ovinos por plantas desse grupo, sob condições naturais, é muito rara. São descritos apenas alguns surtos de intoxicação por Mascagnia rigida na Paraíba (Vasconcelos et al. 2008) e por A. exotropica no Rio Grande do Sul (Bandarra et al. 2007).

Embora a intoxicação criminosa por MF deva ser considerada em casos de morte súbita de ovinos, caprinos e coelhos, acreditamos que sua ocorrência seria menos provável, pois a comercialização desse composto é proibida no país (Brasil 1997). Contudo, sabe-se que este composto ainda é ilegalmente comercializado por ambulantes (Apevisa 2009) e que, se armazenado sob condições adequadas, a sua toxidez é mantida por décadas (Eisler 1995).

Não obstante, a utilização da acetamida no tratamento da intoxicação por plantas que causam "morte súbita", deve ser vista com reserva, uma vez que, para ser efetiva, a droga deveria ser administrada algumas horas antes ou logo após o animal ter sido intoxicado, o que, na prática, para a grande maioria dos casos, é inviável. Apenas para animais que evidenciam uma evolução mais protraída, a droga poderia ter alguma utilidade. Por outro lado, em carnívoros e humanos intoxicados por MF, nos quais o quadro clínico é de evolução mais longa, o uso de acetamida deveria ser incentivado, embora outras substâncias como: barbitúricos (Tourtellette \& Conn 1950), reserpina (Huang et al. 1980), tiossulfato de Mg (Pereira \& Pereira 2005), carvão ativado mais colestipol (Wickstrom et al. 1998), também tenham demonstrado algum efeito benéfico.

\section{REFERÊNCIAS}

Apevisa 2009. Apevisa apreende 302 frascos com raticida ilegal. Disponível em <http://www.saude.pe.gov.br/noticias.php?codigo=1066\&pagi na=2\&publicar=1> Acesso em 20 fev. 2009.

Bandarra P.M., Colodel E.M., Raymundo D.L., Pedroso P.M.O, Borba M.R. \& Driemeier D. 2007. Intoxicações em ruminantes por Mascagnia sp. no Rio Grande do Sul diagnosticadas no Setor de Patologia Veterinária (SPV-UFRGS). XII Encontro Nacional de Patologia Veterinária, Campo Grande, MS. (CD-ROM)

Barbosa J.D., Oliveira C.M.C., Tokarnia C.H. \& Riet-Correa F. 2003. Comparação da sensibilidade de bovinos e búfalos à intoxicação por Palicourea marcgravii (Rubiaceae). Pesq. Vet. Bras. 23(4):167-172.

Brasil 1997. Portaria no 321, de 28 de julho de 1997. Normas Gerais para Registro de Desinfetantes Domissanitários. Disponível em <http:// www.pragas.com.br/legislacao/bancodedados/port321-97.php> Acesso em 15 fev. 2009.

Consorte L.B., Peixoto P.V. \& Tokarnia C.H. 1994. Intoxicação experimental por Pseudocalymma elegans (Bignoniaceae) em ovinos. Pesq. Vet. Bras. 14(4):123-133.

Cunha L.C. 2008. Avaliação dos efeitos tóxicos de Mascagnia rigida em ratos: estudo anatomopatológico, comparação entre metodologias cromatográficas para detecção do fluoroacetato de sódio. Dissertação de Mestrado em Patologia Experimental e Comparada, Universidade de São Paulo, SP. 100p. 
Döbereiner J. \& Tokarnia C.H. 1959. Intoxicação de bovinos pela "erva-de-rato" (Palicourea marcgravii St. Hil.) no vale do Itapicuru, Maranhão. Arqs Inst. Biol. Anim., Rio de J., 2:83-91.

Döbereiner J., Peixoto P.V. \& Tokarnia C.H. 1984. Intoxicação experimental por Arrabidaea bilabiata (Bignoniaceae) em coelhos. Pesq. Vet. Bras. 4(3):89-96.

Egyed M.N. \& Schultz R.A. 1986. The efficacy of acetamide for the treatment of experimental Dichapetalum cymosum (gibflaar) poisoning in sheep. Onderstepoort J. Vet. Res. 54:231-234.

Eisler R. 1995. Sodium monofluoroacetate (1080) hazards to fish, wildlife, and invertebrates: a synoptic review. Contam. Hazard Review 30.

Gava A., Cristiani J., Branco J.V., Dalmo S.N., Mondadori A.J. \& Souza R.S. 1998a. Mortes súbitas em bovinos causadas pela ingestão de Mascagnia sp. (Malpighiaceae), no Estado de Santa Catarina. Pesq. Vet. Bras. 18(1):16-20.

Goncharov N.V., Jenkins R.O. \& Radilov A.S. 2005. Toxicology of fluoroacetate: A review, with possible directions for therapy research. J. Appl. Toxicol. 26(2):148-161.

Górniak S.L., Palermo-neto J. \& Spinosa H.S. 1994. Effects of acetamide on experimentally-induced Palicourea marcgravii (St. Hil) poisoning in rats. Vet. Human Toxicol. 36(2):101-102.

Helayel M.A., França T.N., Seixas J.N., Nogueira V.A., Caldas S.A. \& Peixoto P.V. 2009. Morte súbita em bovinos causada pela ingestão de Pseudocalymma elegans (Bignoniaceae) no município de Rio Bonito, RJ. Pesq. Vet. Bras. 29(7):498-508.

Huang T.Y., Pang X.Q. \& Ch'ang H.L. 1980. Prophylactic effect of reserpine in cardiac failure caused by monofluoroacetic acid derivatives. Acta Pharmacol. Toxicol. 47:78-80.

Jabour F.F., Sexas J.N., Tokarnia C.H. \& Brito M.F. 2006. Variação da toxidez de Arrabidaea bilabiata (Bignoniaceae) em resposta a coelhos. Pesq. Vet. Bras. 26(3):171-176.

Krebs H.C., Kemmerling W. \& Habermehl G. 1994. Qualitative and quantitative determination of fluoroacetic acid in Arrabidea bilabiata and Palicourea marcgravii by F-NMR spectroscopy. Toxicon 32:909-913.

Nogueira V.A., França T.N., Peixoto T.C., Caldas S.A., Armién A.G. \& Peixoto P.V. 2010. Intoxicação experimental por monofluoroacetato de sódio em bovinos: aspectos clínicos e patológicos. Pesq. Vet. Bras. 30(7):533-540.

Oliveira M.M. 1963. Chromatographic isolation of monofluoroacetic acid from Palicourea marcgravii St. Hil. Experientia 19(11):586-587.

Oliveira C.M.C., Barbosa J.D., Macedo R.S.C., Brito M.F., Peixoto P.V. \& Tokarnia C.H. 2004. Estudo comparativo da toxidez de Palicourea juruana (Rubiaceae) para búfalos e bovinos. Pesq. Vet. Bras. 24(1):27-30.
Peixoto T.C., Nogueira V.A., Coelho C.D., Veiga C.C.P., Peixoto P.V. \& Brito M.F. 2010. Aspectos clínico-patológicos e laboratoriais da intoxicação experimental por monofluoroacetato de sódio em ovinos. Pesq. Vet. Bras. 30(12):1021-1030.

Peixoto P.V., Nogueira V.A., França T.N., Peixoto T.C., Döbereiner J. \& Tokarnia C.H. 2011a. Relationship between a peculiar form of hydropic-vacuolar degeneration of the distal convoluted tubules, monofluoroacetate poisoning, and plants that cause "sudden death" in Brazil, p.365-372. In: Riet-Correa F., Pfister J., Schild A.L. \& Wierenga T. (Eds), Poisoning by Plants, Mycotoxins, and related Toxins. CABI, London.

Peixoto T.C., Nogueira V.A., Caldas S.A., França T.N. \& Peixoto P.V. 2011b. Efeito protetor da acetamida em bovinos indica ácido monofluoroacético como princípio tóxico de Palicourea marcgravii. (Submetido para publicação)

Peixoto T.C., Caldas S.A., Iglesias L., Catunda junior F.E.A., França T.N. \& Peixoto P.V. 2011c. Efeito protetor da acetamida sobre a intoxicação experimental por monofluoroacetato de sódio e plantas que causam morte súbita em ratos. (Submetido para publicação)

Pereira N.A. \& Pereira S.M.N. 2005. Contribuição ao estudo de plantas tóxicas e seus antagonistas: erva-de-rato, a Rubiaceae, Palicourea marcgravii. Revta Bras. Farm. 86(3):109-11.

Tavares M.I., Rezende A.M.L. \& Döbereiner J. 1974. Intoxicação experimental por Pseudocalymma elegans em coelhos e cobaias. Pesq. Agropec. Bras. 9:91-94.

Tokarnia C.H., Döbereiner J., Canella C.F.C. \& Guimarães D.J. 1969. Intoxicação experimental por Pseudocalymma elegans (Vell.) Kuhlm. em bovinos. Pesq. Agropec. Bras. 4:195-204

Tokarnia C.H., Peixoto P.V. \& Döbereiner J. 1993. Intoxicação experimental por Pseudocalymma elegans (Bignoniaceae) em caprinos. Pesq. Agropec. Bras. 13(1/2):35-39.

Tokarnia C.H., Döbereiner J. \& Peixoto P.V. 2000. Plantas Tóxicas do Brasil. Helianthus, Rio de Janeiro. 310 p.

Tourtellotte W.W. \& Coon J.M. 1950. Treatment of fluoroacetate poisoning in mice and dogs. J. Pharmacol. Expl Therapeutics 101:82-91

Vasconcelos J.S., Riet-Correa F., Dantas A.F.M., Medeiros R.M.T., Galiza G.J.N., Oliveira D.M. \& Pessoa A.F.A. 2008. Intoxicação por Mascagnia rigida (Malpighiaceae) em ovinos e caprinos. Pesq. Vet. Bras. 28(10):521-526.

Wickstrom M., Ataria J.M., O`Halloran K. \& Eason C.T. 1998. Development of antidotes for improved treatment of 1080 toxicosis. Landcare Research Contract Report to the Animal Health Board. Abstract available from <http://tbfree.ahb.org.nz> Acesso em 2010. 